\title{
Laparoscopic Trans-Abdominal Retromuscular (TARM) Repair for Ventral Hernia: A Novel, Low-Cost Technique for Sublay and Posterior Component Separation
}

\author{
Ashwin A. Masurkar ${ }^{1}$
}

Published online: 26 November 2019

(C) The Author(s) 2019

\begin{abstract}
Background The complications of intraperitoneal onlay mesh repair for ventral hernia has favored sublay mesh placement like open Rives-Stoppa repair (ORS). There was a need for low-cost laparoscopic trans-abdominal repair using a polypropylene mesh (PPM) with sublay, midline closure and addition of posterior component separation (PCS) by transversus abdominis release (TAR).

Methods The techniques used three or six operating ports with triangulation. After adhesiolysis, a transverse incision was made on the peritoneum (P) and posterior rectus sheath (PRS). The retromuscular space was developed by raising a P-PRS flap. Midline closure was performed with No. 1 polydioxanone, and a PPM was placed in sublay, followed by closure of defect and P-PRS incision. For large hernias with divarication; myo-fascial medialization using PCS-TAR aided low-tension midline closure.

Results Eighty-nine patients were operated from 2010 to 2019, 26 primary ventral; 63 incisional; and 22 recurrent hernias. Of the primary, 21 were umbilical, one Spigelian and four epigastric hernias. The incisional group had 57 patients with lower midline scars (C-section 25, open tubal ligation 15, abdominal hysterectomy 17), five lateral (appendicectomy), one post-laparotomy. The mean age, male/female sex ratio and BMI were 41.23 years, 1:10.1 and $29.2 \mathrm{~kg} / \mathrm{m}^{2}$, respectively. Mean defect and mesh area were $110 \mathrm{~cm}^{2}$ and $392 \mathrm{~cm}^{2}$. Mean operating time was $192 \mathrm{~min}$. Conversion to open, mesh infection and recurrence rates were $3.4 \%, 1.1 \%$ and $5.62 \%$.

Conclusion Laparoscopic TARM with PPM in sublay avoids mesh-bowel contact. It provides midline closure and PCS-TAR within the same port geometry with results comparable with ORS.
\end{abstract}

Presented by the author at the hernia video session at the World Congress of Endoscopic Surgery hosted by SAGES (Society of American Gastrointestinal and Endoscopic Surgeons) in April 2018, at Seattle, USA.

Ashwin A. Masurkar

ashwinmasurkar@gmail.com;

https://www.masurkarhospital.com

1 Masurkar Hospital, RS No 136, Naka no 1, (District Belgaum), Gokak, Karnataka 591307, India

\section{Introduction}

The complications of intraperitoneal onlay mesh (IPOM) repair for ventral hernias have favored sublay mesh placement like the open Rives-Stoppa repair (ORS) [1]. Midline closure has been recommended for restoration of the biomechanics of the abdominal wall [2]. Based on these concepts, several techniques have been developed; like trans-peritoneal sublay [3] and trans-extra-peritoneal (eTEP) repair [4]. The latter additionally combined midline closure and posterior component separation (PCS). There was however, a need for a low-cost laparoscopic (transabdominal) repair using a polypropylene mesh (PPM) with 
sublay, midline closure [2] and the optional addition of PCS by transversus abdominis release (TAR) [5]. This study was aimed at developing this technique using a familiar and ergonomic port geometry.

\section{Surgical techniques}

Patients with uncomplicated ventral hernias were included. All patients were operated under general anesthesia, in a modified lithotomy position. A urinary catheter and a nasogastric tube were passed. A high-definition camera system and two monitors were used (Fig. 1).

Peritoneal access was via open Hasson's technique using a $10-\mathrm{mm}$ trocar. The operation table was flexed for wider instrument manipulation angles. Based on location, size of hernia and associated rectus abdominis divarication, three techniques were developed as follows.

\section{Technique 1: Three-port TARM (Figs. 1, 2)}

This technique was devised for supra-umbilical, infraumbilical and lateral hernias. Three trocars were used: one $10 \mathrm{~mm}$ and for telescope and two 5-mm trocars as working ports (Fig. 1, labeled T1, W1 and W2) placed in the upper abdomen for infra-umbilical and lateral hernias; and in the lower abdomen (Fig. 3, labeled T2, W3 and W4) for epigastric hernias, respectively.

Working ports were placed medial to the linea semilunaris (LS), preoperatively marked by ultrasonography.

After adhesiolysis and reduction of contents of the hernial sac, the defect was assessed. Using electrosurgery or harmonic scalpel, a 6-8 cm long transverse incision was made on the peritoneum $(\mathrm{P})$ and posterior rectus sheath

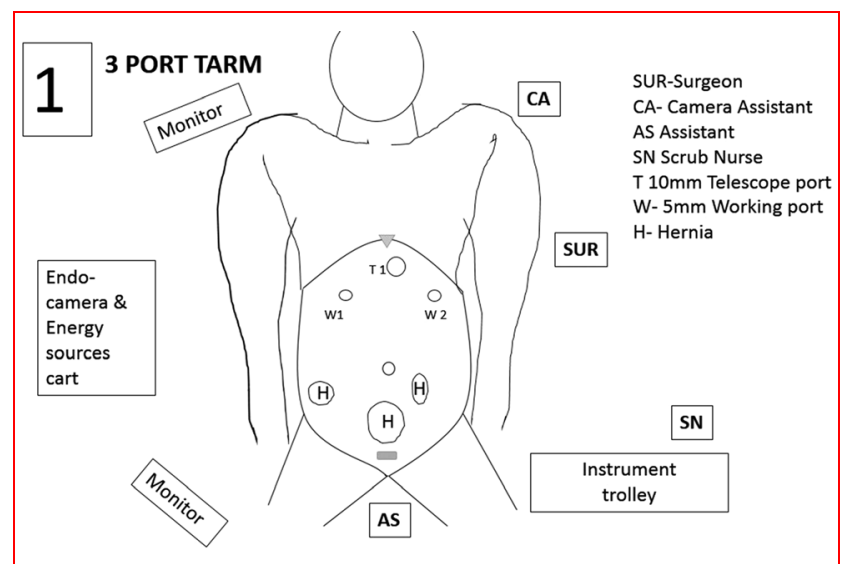

Fig. 1 Operating room set up \& port positions: 3 PORT TARM

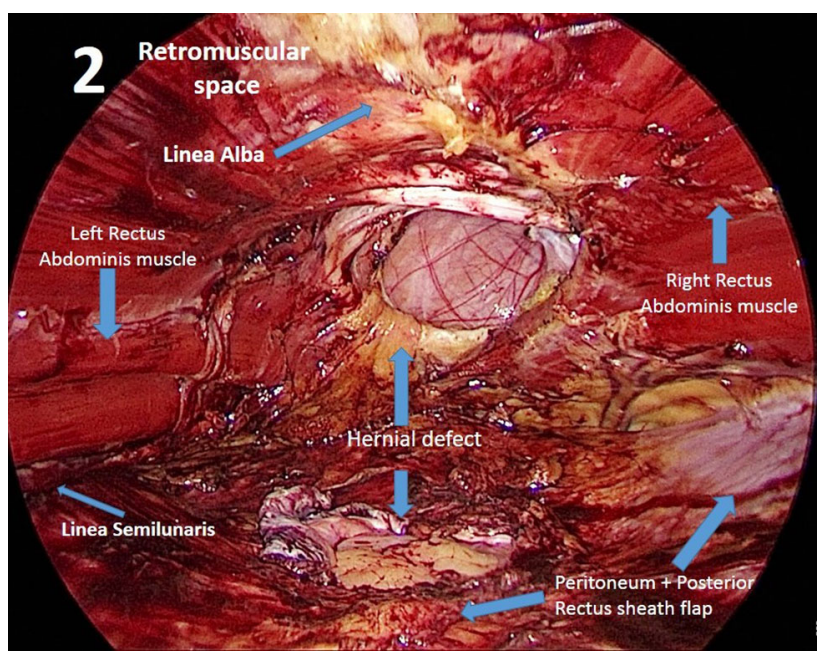

Fig. 2 Infra-umbilical retromuscular space as viewed from camera port in epigastrium (T1)

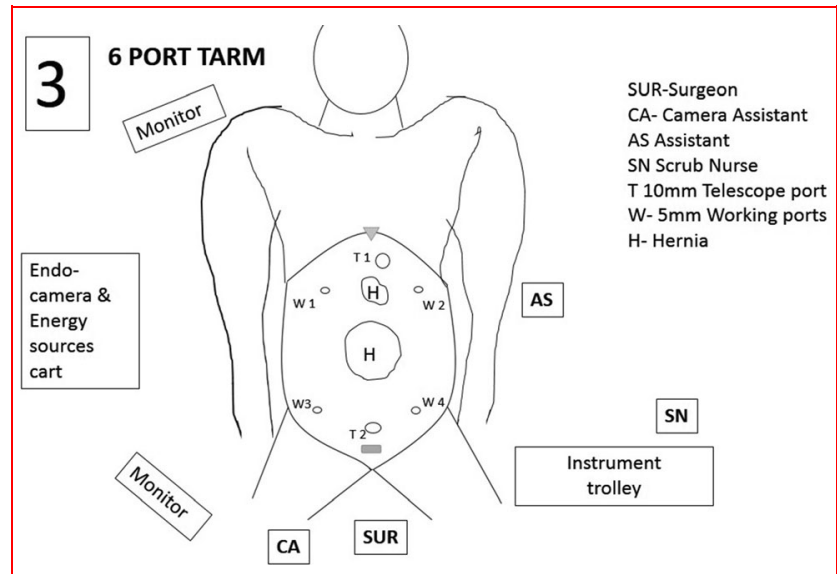

Fig. 3 Operating room set up \& port positions: 6 PORT TARM \& TARM-TAR

(PRS), underlying the rectus abdominis muscle, 5-6 cm proximal to the defect. The retromuscular space was developed by raising a flap of P-PRS, $8 \mathrm{~cm}$ beyond the hernial defect, with careful preservation of epigastric vessels, neurovascular bundles at the LS and linea alba (LA). The intra-abdominal pressure was then reduced to $8 \mathrm{~mm} \mathrm{Hg}$. Midline closure [2] was performed with a running suture of No. 1 polydioxanone (PDS) passing through rectus abdominis muscles, anterior rectus sheath and LA. A medium-weight microporous PPM with wide overlap was parked into the retromuscular space. The hernial defect and initial P-PRS incision were closed using No. 0 PDS, followed by desufflation and port closure. 


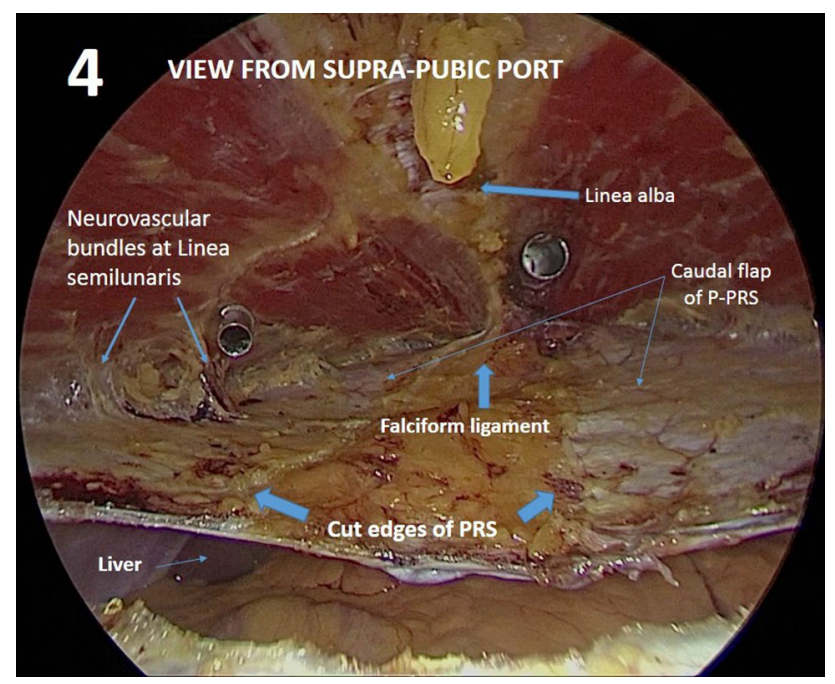

Fig. 4 Supra-umbilical retromuscular space as viewed from suprapubic camera port (T2)

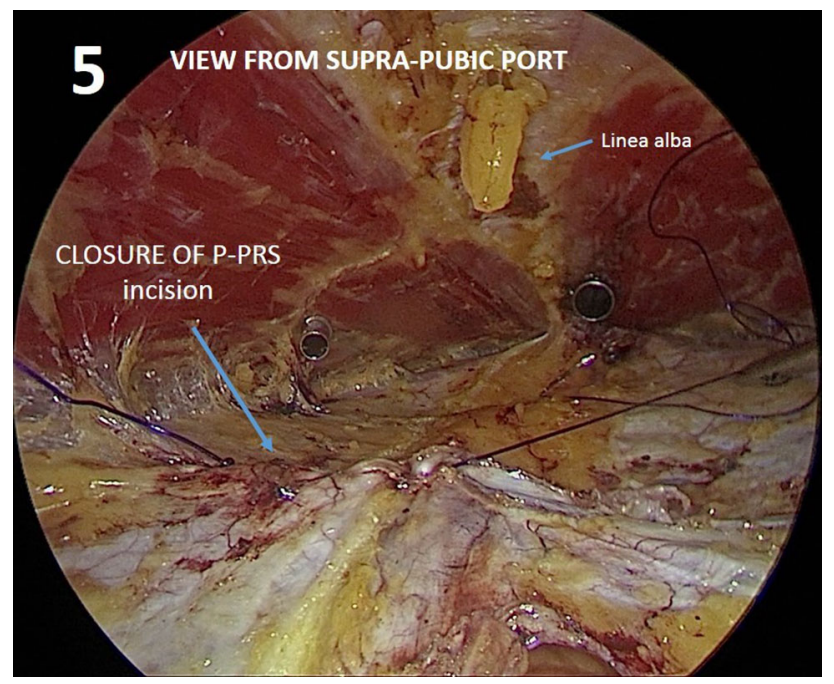

Fig. 5 Closure of peritoneum-posterior rectus sheath incision

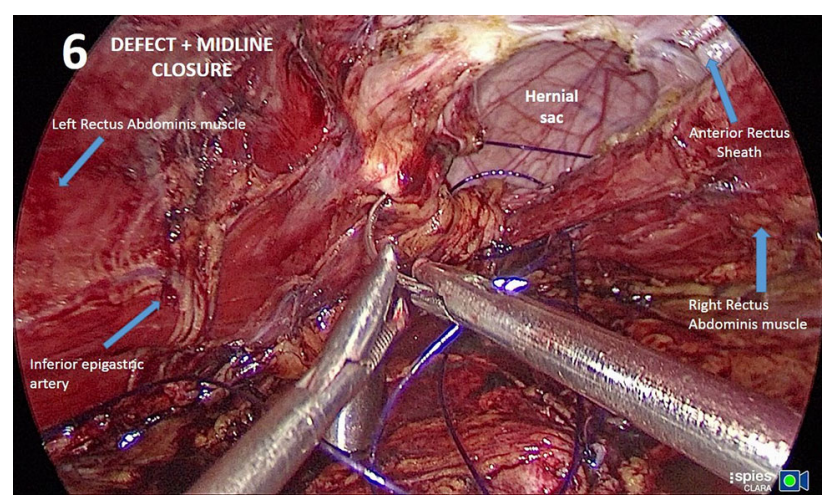

Fig. 6 Midline and hernial defect closure

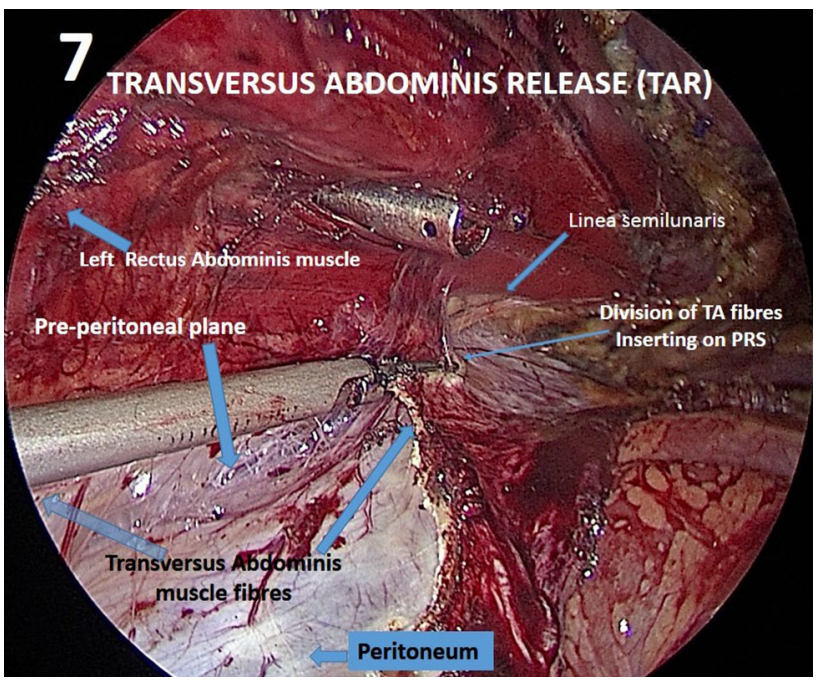

Fig. 7 Transversus abdominis release

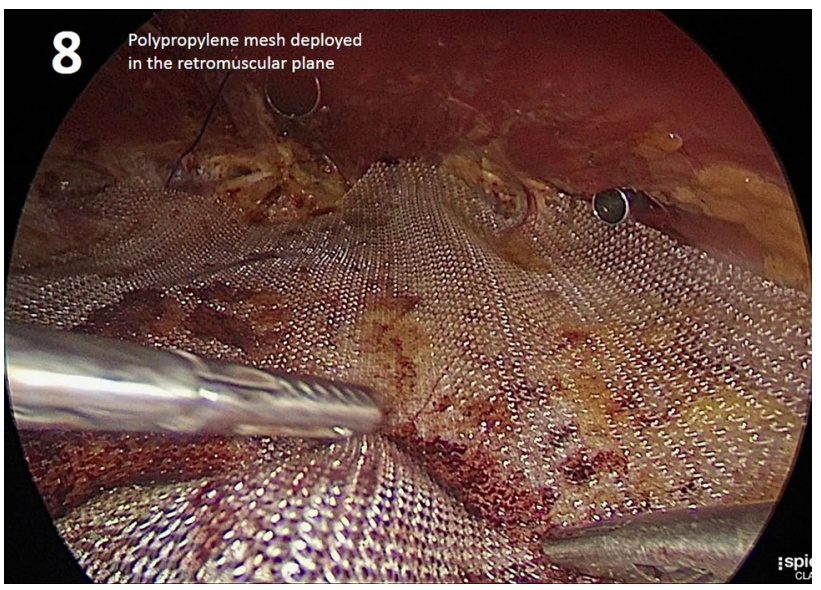

Fig. 8 Mesh deployment into the retromuscular space

Technique 2: six-port TARM (Figs. 1, 2, 3, 4, 5, 6, 7 and 8)

This technique was devised to repair large hernias, particularly umbilical, para-umbilical, multiple defects and divarication of recti. Six ports were used to raise two P-PRS flaps. The first trocar was inserted into the epigastrium with two $5 \mathrm{~mm}$ working ports (Fig 1 labeled T1, W1 and W2) medial to LS. A transverse incision was made on the P-PRS, midway between the xiphisternum and symphysis pubis. The retro-rectus space was developed inferiorly till the symphysis pubis and laterally till the LS. Three more ports were inserted into the lower abdomen to lie in the retromuscular space (Fig. 3 labeled T2, W3 and W4): one $10-\mathrm{mm}$ camera port in the supra-pubic area and 
two 5-mm working ports. The telescope was then shifted to the supra-pubic port. With the surgeon standing between the patient's legs, an upper flap of P-PRS was mobilized till the level of the xiphisternum. The initially inserted three trocars were withdrawn into the retromuscular space. The intra-abdominal pressure was reduced to $8 \mathrm{~mm} \mathrm{Hg}$, followed by closure of defects and the initial transverse incision on P-PRS (Fig. 5) with running suture of PDS. The defect in the anterior sheath, midline and divarication was approximated (Fig. 6).

Finally, a PPM of maximum size (usually $15 \times 30$ or $20 \times 30 \mathrm{~cm}$ ) was inserted. It was spread out between one LS and the other and from xiphisternum to pubic symphysis (Fig. 8), without fixation, to achieve complete reinforcement of the visceral sac like ORS [1].

Technique 3: TARM-TAR: addition of transversus abdominis release (TAR) [5] to one of the above

This technique was devised for large hernias with wide divarication, or when midline closure was under tension as observed during intra-operative assessment. PCS by TAR was added to either of the previous techniques providing myo-fascial release. The transversus abdominis (TA) muscle fibers were identified in the upper abdomen inserting into the PRS medial to the LS. The muscle was incised (Fig 7) followed by dissection into the pre-peritoneal space, to provide reduced tension for midline closure. Next, a $30 \times 30 \mathrm{~cm}$ PPM was parked into the retromuscular space. A second $15 \times 15 \mathrm{~cm}$ mesh was placed in the lower abdomen in diamond-shaped orientation, to form a mesh "home plate."

Dilute gentamicin was sprayed to prevent mesh infection. Suction drains were placed over the mesh, and compression dressings were applied to reduce seroma formation.

\section{Results}

Eighty-nine patients with uncomplicated hernias were operated from February 2010 to February 2019. Twentysix primary ventral, 63 incisional and 22 recurrent hernias were treated. Of the primary, 21 were umbilical, one Spigelian and four epigastric hernias. In the incisional group, 57 were of lower midline scars (LSCS 25, open tubal ligation 15, abdominal hysterectomy 17), five lateral (appendectomy scars) and one lower midline laparotomy incision. The mean age, male/female sex ratio and BMI were 41.23 years, $1: 10.1$ and $29.2 \mathrm{~kg} / \mathrm{m}^{2}$, respectively.
Mean defect area was $110 \mathrm{~cm}^{2}$ (range 176-12), and mesh area was $392 \mathrm{~cm}^{2}$. The mean operating time, blood loss and length of stay were $192 \mathrm{~min}, 35.2 \mathrm{ml}$ and 5 days, respectively. Three were converted $(3.4 \%)$ to ORS due to dense bowel adhesions. Technique-wise case distribution was 47, 35 and 7 for three-port TARM, six-port TARM and TARM-TAR, respectively.

One mesh infection $(1.1 \%)$ presented at 20 days postdischarge and was treated by explantation. The hernia recurred after 6 months and was treated by ORS. A mean follow-up of 58.71 (range 108 to 4) months revealed five late recurrences $(5.62 \%)$ for lower abdominal incisional hernias, in the three-port TARM group. Laparoscopy revealed P-PRS suture-line disruption at the caudal border of mesh, where the divarication had remained unrepaired and unsupported by mesh. All were treated by ORS. No recurrence was noted in the six-port or TARM-TAR group owing to extensive mesh cover. Three patients successfully underwent laparoscopy for other pathologies (one hysterectomy and two appendectomy); and revealed unhindered peritoneal access, favorable mesh integration, with only minimal omental adhesions.

\section{Comments}

Laparoscopic TARM using PPM was found to be effective for repairing small, medium and selected large hernias. Sublay mesh placement avoided mesh-bowel contact. Midline closure and PCS-TAR [5] were added within the same port positions. The latter resembles the port geometry of trans-abdominal pre-peritoneal (TAPP) repair for inguinal hernia, and is familiar to most surgeons. The larger working space and clear anatomy provide easy understanding and reproducibility. Standard laparoscopic instrumentation was used. Intraperitoneal composite meshes, balloon dissectors [4] and robotic arms were not needed. Further cost reduction could be achieved by using electrosurgical dissection instead of harmonic scalpel. The approximate cost comparison (local prices in US Dollars) for disposables, viz. $15 \times 15 \mathrm{~cm}$ mesh+fixation devices+sutures; for IPOM, eTEP, TARM (using electrosurgery) and ORS is $685,305,81$ and 75 respectively. As regards cost of disposables, TARM is marginally more expensive than ORS. Midline closure ensured restoration of biomechanics of the abdominal wall [2]. Myo-fascial medialization for low-tension closure could be achieved via PCS-TAR [5] within the same ports. With outcomes comparable with ORS, these results support further evaluation of the technique in a large multicenter trial. 
Funding None.

Data availability Available on SAGES YouTube ${ }^{\circledR}$ channel "SAGES TV" https://youtu.be/N6U1izzlc8k and author's YouTube ${ }^{\circledR}$ channel https://youtu.be/vxcVxv4S3r8.

\section{Compliance with ethical standard}

Conflict of interest The authors declare that they have no conflict of interests.

Ethical approval The study was approved by the hospital ethics.

Informed consent Informed consent for procedure and consent for review of patient data were taken from all the participating patients.

Open Access This article is distributed under the terms of the Creative Commons Attribution 4.0 International License (http://crea tivecommons.org/licenses/by/4.0/), which permits unrestricted use, distribution, and reproduction in any medium, provided you give appropriate credit to the original author(s) and the source, provide a link to the Creative Commons license, and indicate if changes were made.

\section{References}

1. Stoppa RE (1989) The treatment of complicated groin and incisional hernias. World J Surg 13(5):545-554. https://doi.org/ 10.1007/bf01658869

2. Rosen MJ, Fatima J, Sarr MG (2010) Repair of abdominal wall hernias with restoration of abdominal wall function. J Gastrointest Surg 14(1):175-185. https://doi.org/10.1007/s11605-009-0981-9

3. Schroeder AD, Debus ES, Schroeder M, Reinpold WM (2013) Laparoscopic transperitoneal sublay mesh repair: a new technique for the cure of ventral and incisional hernias. Surg Endosc 27(2):648-654. https://doi.org/10.1007/s00464-012-2508-9

4. Belyansky I, Daes J, Radu VG, Balasubramanian R, Reza Zahiri H, Weltz AS, Sibia US, Park A, Novitsky Y (2018) A novel approach using the enhanced-view totally extraperitoneal (e TEP) technique for laparoscopic retromuscular hernia repair. Surg Endosc 32(3):1525-1532. https://doi.org/10.1007/s00464-017$5840-2$

5. Novitsky YW, Elliott HL, Orenstein SB, Rosen MJ (2012) Transversus abdominis muscle release: a novel approach to posterior component separation during complex abdominal wall reconstruction. Am J Surg 204(5):709-716. https://doi.org/10. 1016/j.amjsurg.2012.02.008

Publisher's Note Springer Nature remains neutral with regard to jurisdictional claims in published maps and institutional affiliations.

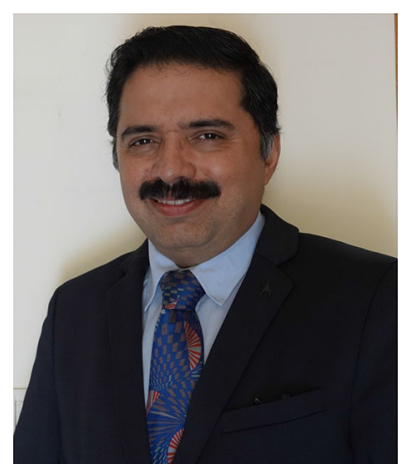

Dr Ashwin Masurkar was born and raised in Mumbai, India. He obtained his medical degree and surgical training from the University of Mumbai. He was trained in minimally invasive surgery during his residency days, and he developed it as his core interest. Being passionate about rural health care, he runs a private surgical hospital in Gokak, a small town in South India. His area of focus is laparoscopic hernia repair and abdominal wall reconstruction.

He travels all over the country to train surgeons in this specialty. His hobbies include playing the guitar, singing and farming. His twitter handle is @dr_masurkar. 